\title{
Mental capacity assessment: a descriptive, cross- sectional study of what doctors think, know and do
}

\section{Abstract}

Background The Mental Capacity Act (MCA) (2005) was enacted in 2007 in England and Wales, but the assessment of mental capacity still remains an area of professional concern. Doctors' compliance with legal and professional standards is inconsistent, but the reasons for poor compliance are not well understood. This preliminary study investigates doctors' experiences of and attitudes toward mental capacity assessment (MCAx).

Methods This is a descriptive, cross-sectional study where a two-domain, study-specific structured questionnaire was developed, piloted and digitally disseminated to doctors at differing career stages employed in a large, multi-site National Health Service Trust in London over 4 months in 2018. Descriptive statistics and frequency tables adjusted for missing data were generated and secondary analysis was conducted.

Results Participants $(n=92)$ were predominantly UK trained $(82 \%)$, female $(58 \%)$ and between the ages of 30 and 44 years (45\%). Less than half (45\%) of the participants reported receiving formal MCAx training. Only one-third (32\%) of the participants self-rated themselves as very competent $(29 \%)$ or extremely competent $(4 \%)$. Self-reported MCA confidence was significantly affected by career stage with Consultants with over 10 years of experience reporting lowest confidence $(p=0.001)$.

Conclusions This study describes significant variation in practice by doctors and low selfconfidence in the practice of MCAx. These results raise concerns that MCAx continues to be inconsistently performed by doctors despite appropriate awareness of the law and professional guidance on best practice.

\section{Introduction}

Although the introduction of the Mental Capacity Act 2005 (MCA) in England and Wales was welcomed, its implementation has proved challenging for doctors and other healthcare professionals. ${ }^{1(\mathrm{p} .23)}$ Variable mental capacity assessment (MCAx) practice by healthcare professionals has undermined its impact. .2 A post-legislative review of the Act by the House of Lords highlighted inconsistent and inadequate application of the processes within it. 1 Implementation of the Act has not met the expectations due to low awareness and poor understanding with little integration into existing care pathways impacting the confidence of medical staff to discuss issues or complete documentation. $\underline{\underline{2-5}}$ These factors combined with a paternalistic safety culture can have profound consequences for vulnerable patients, their caregivers and family. 1 Yet, the reasons for poor application of the MCA are not well understood. This preliminary study aimed to explore doctors' experiences of and attitudes toward MCAx. 


\section{Background}

The Mental Capacity Act (2005)6 was born out of a desire to codify existing common law in order to provide a clearer means to judge the decision-making capacity of vulnerable people. The MCA created the presumption of capacity and outlines the test required to establish whether someone lacks the capacity to make a specific decision. It provides a legislative framework designed to cover all decisions especially those concerning personal welfare, healthcare and financial affairs.7 It is accompanied by a written Code of Practice 8 and was described as 'a very significant and progressive piece of legislation, with the potential to transform lives.' 1(p.23) The Act outlines the components of mental capacity, when assessments ought to be considered and how they should be carried out.

Considerable uncertainty about how best to implement the Act in practice has undermined the ethos of empowerment the Act intended to promote. The House of Lords concluded that despite being held in high regard by health and social care professionals, the Act was not properly embedded in practice. 1 The Act was described as being applied as an 'optional add-on' with no place in the core working lives of health and social care workers. 1 This echoes the views of healthcare staff in neurology and palliative care who reported poor confidence in applying the Act due to a lack of training.9

Scant attention has been paid to examining healthcare professionals' experiences of MCAx and there is a particular dearth of evidence pertaining to doctors. Limited previous studies have examined mixed groups of healthcare professionals including some doctors, mainly in the context of dementia and psychiatric care but the numbers are small.9-11 This is concerning as doctors, particularly the most senior members of treating medical teams, are the professionals most often responsible for performing MCAx in the secondary and tertiary care settings.2 8(p.54) This exploratory study aimed to address this gap by examining the attitudes and experiences of doctors to mental capacity assessment using a survey.

\section{Methods}

Using a descriptive, cross-sectional design, a two-domain, study-specific structured questionnaire, The Clinician Mental Capacity Assessment (CMCA), was designed and piloted with healthcare 
professionals and carers in accordance with established methodology. ${ }^{12}$ The primary aim of the study was to describe the attitudes of doctors to mental capacity assessment in secondary and tertiary care. The description of the experience of doctors and the exploration of relationships between their attitudes and experience were the secondary aims. This study was registered with the local Data Protection Registration Service and was reviewed by the local Research Ethics Committee and approved as a service evaluation.

\section{Sample}

The questionnaire was electronically disseminated and anonymously self-administered by participants. Doctors employed at a single multi-site London National Health Service (NHS) Trust were sampled. As this particular NHS Trust was a secondary and tertiary care centre it was not possible to obtain a sample inclusive of primary care doctors. All consultants and junior doctors on the electronic mail distribution list of a large London NHS Trust consisting of multiple secondary and tertiary hospitals were invited to participate over a 4-month period beginning in January 2018. Reminder emails were sent once midway and once near the end of the recruitment period.

\section{Questionnaire design and rationale}

A questionnaire was designed to facilitate the scalable collection of unambiguous quantitative data. ${ }^{12}$ Following extensive piloting the CMCA questionnaire, which assesses two domains, attitude, and experience, was finalised.

Domain 1, experience, was assessed with single best answer questions with an aim to characterise the experience and training history of participants. Data on variables related to: MCAx frequency in the previous 12 months; familiarity with professional guidelines; formal training received; time taken to perform MCAx; and confidence in recognising when MCAx are required or should be queried.

Domain 2, attitudes, explored the thoughts of participants using Likert scales anchored to a clinical vignette concerning discharge arrangement decisions as a point of reference. ${ }^{4}{ }^{13}$ The feelings of participants toward performing MCAx in general were also captured. Self-reported questionnaires sometimes yield less reliable results on competence, however, the clinical vignette served as an indicator of how participants might use their knowledge to exercise their judgement as they would do in practice. Demographic data were collected: age group, gender identity, career stage, clinical speciality and country of primary medical qualification. 
The questionnaire was piloted in three healthcare professional groups; doctors, mental health nurses and speech and language therapists, to ensure that the questions were clear and flowed in a logical fashion. ${ }^{12}$ It was assumed that $50 \%$ of potential participants were likely to have performed a mental capacity assessment at least once in their career, therefore, it was calculated that the study would require 97 participants for estimating the expected proportion with $10 \%$ absolute precision and $95 \%$ confidence.

\section{Materials and resources}

The Opinio online survey platform and IBM SPSS Statistics for Windows, Version 25.0 were used in accordance with the terms of license. ${ }^{14} 15$

\section{Statistical analysis}

Data received from Opinio were categorical in nature and were automatically assigned respondent identification codes by the platform. Opinio produced an electronic data set which was reviewed using IBM SPSS Statistics for Windows. ${ }^{14}$ These data were coded, cleaned and examined for coding errors to ensure consistency. There were relatively few missing values and an imputation of these values was considered. However, as the potential gain in sample size from imputation would not have increased the power substantially, it was not deemed necessary to impute missing values in the data set.

A random sample of five individual responses was examined and verified against source data to ensure consistent coding. Frequency tables of a random sample of $10 \%$ of cases were also reviewed to ensure valid coding. Descriptive statistics and frequency tables were then produced and based on these preliminary results secondary analysis was conducted. This comprised MannWhitney and Kruskal-Wallis tests to assess differences in the attitudes and experiences seen in different groups of doctors. Fisher's exact tests were also conducted to compare the demographics of the participants who completed the questionnaire and those who did not. A significance level of 0.05 was used for hypothesis testing.

\section{Results}

In January 2018, an invitation email was sent to 1634 doctors on the Trust distribution list. There were 607 failed email transmissions therefore 1027 doctors were contacted. One hundred and six doctors responded (ie, clicked on the invitation link to the questionnaire) yielding a response rate of 
$10 \%$. Three respondents did not provide consent to participate and therefore did not attempt the questionnaire. Eleven respondents provided consent but did not attempt any questions and were excluded from the analysis. As all questionnaire responses were anonymous it was not possible to characterise the 14 respondents who did not attempt the questionnaire. Ninety-two doctors attempted the questionnaire and there was no difference between the demographic variables previously described when those who completed the questionnaire $(90 \%, 83 / 92)$ and those who did not $(10 \%, 9 / 92)$ were compared using Fisher's exact tests (table 1$)$.

\section{Table 1}

Summary of participant demographics. Includes Fisher's exact tests of significance comparing participants who completed all questions and participants who did not complete all questions.

\begin{tabular}{|c|c|c|c|c|c|c|}
\hline \multirow{3}{*}{\multicolumn{2}{|c|}{ Participant Demographics }} & \multicolumn{4}{|c|}{ Participants } & \multirow{3}{*}{$\begin{array}{c}\text { Completed/Non- } \\
\text { completed } \\
\text { response } \\
\text { comparison } \\
\text { Fisher's exact }\end{array}$} \\
\hline & & \multirow{2}{*}{$\begin{array}{c}\text { Completed } \\
\mathbf{n}\end{array}$} & \multirow{2}{*}{\begin{tabular}{|c|}
$\begin{array}{c}\text { Non- } \\
\text { completed }\end{array}$ \\
$\mathbf{n}$
\end{tabular}} & \multicolumn{2}{|c|}{ Total } & \\
\hline & & & & $\mathbf{n}$ & $(\%)$ & \\
\hline \multirow[t]{5}{*}{ Age } & $18-29$ & 14 & 0 & 14 & 15 & \multirow[t]{5}{*}{$p=0.644$} \\
\hline & $30-44$ & 36 & 5 & 41 & 45 & \\
\hline & $45-54$ & 25 & 3 & 28 & 30 & \\
\hline & $55-64$ & 7 & 1 & 8 & 9 & \\
\hline & $65+$ & 1 & 0 & 1 & 1 & \\
\hline \multirow{4}{*}{$\begin{array}{l}\text { Gender } \\
\text { identity }\end{array}$} & Female & 47 & 6 & 53 & 58 & \multirow[t]{4}{*}{$p=0.782$} \\
\hline & Male & 34 & 3 & 37 & 40 & \\
\hline & Trans & 1 & 0 & 1 & 1 & \\
\hline & Prefer not to disclose & 1 & 0 & 1 & 1 & \\
\hline \multirow{3}{*}{$\begin{array}{r}\text { Country of } \\
\text { Primary } \\
\text { Medical } \\
\text { Qualification }\end{array}$} & United Kingdom & 67 & 8 & 75 & 82 & \multirow[t]{3}{*}{$p=0.335$} \\
\hline & $\begin{array}{l}\text { European Union or European Economic } \\
\text { Area }\end{array}$ & 12 & 0 & 12 & 13 & \\
\hline & $\begin{array}{l}\text { Non-European Union or Non-European } \\
\text { Economic Area }\end{array}$ & 4 & 1 & 5 & 5 & \\
\hline \multirow[t]{6}{*}{ Career stage } & $\begin{array}{l}\text { Recently Qualified - Foundation Training } \\
\text { (FY1 to FY2) }\end{array}$ & 5 & 0 & 5 & 5 & \multirow[t]{5}{*}{$p=0.310$} \\
\hline & $\begin{array}{l}\text { Early Speciality Training - Core Training } \\
\text { (CT1 to ST3) }\end{array}$ & 11 & 3 & 14 & 15 & \\
\hline & $\begin{array}{l}\text { Senior Speciality Training - Higher } \\
\text { Speciality Training (ST4 to CCT) }\end{array}$ & 18 & 1 & 19 & 21 & \\
\hline & $\begin{array}{l}\text { Consultant with less than } 10 \text { years of } \\
\text { experience }\end{array}$ & 16 & 2 & 18 & 20 & \\
\hline & $\begin{array}{l}\text { Consultant with over } 10 \text { years of } \\
\text { experience }\end{array}$ & 31 & 2 & 33 & 36 & \\
\hline & Neurology & 17 & 2 & 19 & 21 & $p=0.978$ \\
\hline
\end{tabular}




\begin{tabular}{|c|c|c|c|c|c|}
\hline \multirow{2}{*}{$\begin{array}{l}\text { Clinical } \\
\text { speciality }\end{array}$} & Anaesthetics & 9 & 1 & 10 & 11 \\
\hline & Geriatric Medicine & 7 & 2 & 9 & 10 \\
\hline \multirow[t]{2}{*}{${ }^{*}$ Top 5} & Haematology & 6 & 0 & 6 & 7 \\
\hline & Obstetrics and Gynaecology & 4 & 2 & 6 & 7 \\
\hline
\end{tabular}

Participants were predominantly UK trained $(82 \%, 75 / 92)$ and female $(58 \%, 53 / 92)$. Age was approximately normally distributed with the majority of doctors ranging in age between 30 and 44 years $(45 \%, 41 / 92)$. Most of the participants were at Consultant career stage $(55 \%, 51 / 92)$. Neurology $(31 \%, 19 / 92)$ was the most common speciality followed by Anaesthetics (11\%, 10/92) and Geriatric Medicine (10\%, 9/92). However, there was high variation in the sample with responses coming from over 20 clinical specialities representing the wide scope of services delivered by the Trust (table 1).

\section{Experience and training}

Most of the participants $(83 \%, 76 / 92)$ had performed a MCAx at least once in their career either independently or as a part of a multidisciplinary team. The majority $(70 \%, 52 / 74)$ of participants who have performed a MCAx prior had done so in the previous 12 months and reported performing MCAx 10 or fewer times in the previous 12 months (62\%, 32/52). Twenty-seven per cent (24/89) of the participants reported not being aware of the professional guidance on MCAx, with less than half $(45 \%, 40 / 89)$ reporting receiving formal training which was conducted both face-to-face and as an eLearning module. When asked about their familiarity with professional guidelines, the majority $(67 \%, 4 / 6)$ of those who did not complete other aspects of the questionnaire indicated that they were not familiar with the said guidelines.

\section{Practice variation}

Although $73 \%$ (65/89) of the participants reported that they were aware of the professional guidance on MCAx, substantial practice variability was reported. Most (43\%, 30/70) reported taking 11 to $20 \mathrm{~min}$ to perform a MCAx. Some participants reported taking as little as 1 to $10 \mathrm{~min}(21 \%, 15 / 70)$ to perform a MCAx while others reported taking 31 to $60 \mathrm{~min}(10 \%, 7 / 70)$. Fewer than half of the participants $(41 \%, 38 / 92)$ reported following local guidelines with 15\% (14/92) admitting to incorrectly judging mental capacity against commonly held beliefs. Additionally, 22\% (19/86) of the participants reported being 'somewhat confident' or 'not at all confident' in recognising when a mental capacity assessment is justified. Half of the participants $(50 \%, 43 / 86)$ have queried the need for an MCAx, with 11\% (9/86) witnessing an MCAx being performed without a clear indication. The most common reason for querying an MCAx was fluctuating capacity (28\%, 26/92) (figure 1). 


\section{Figure 1}

Reasons for querying a mental capacity assessment.

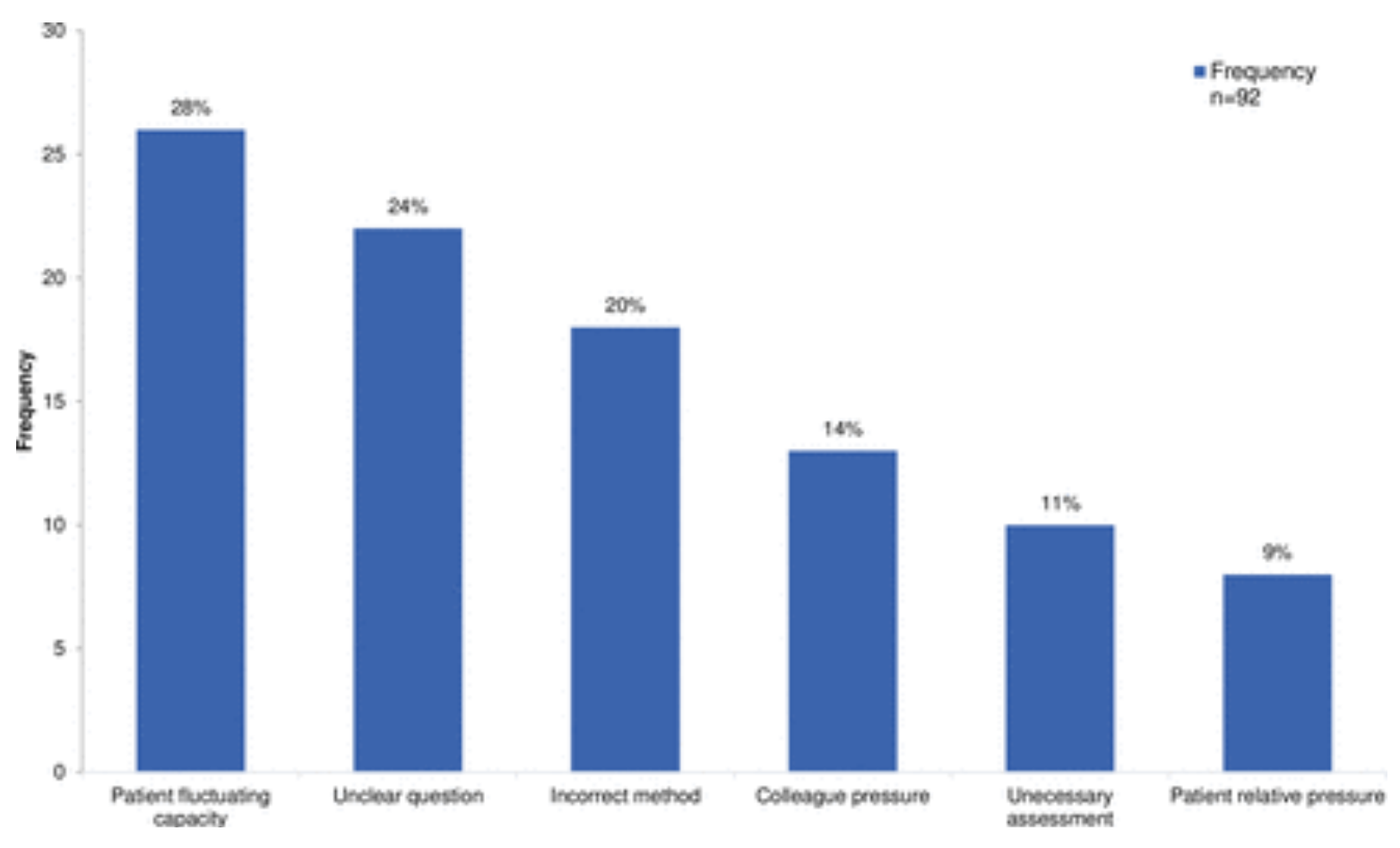

\section{Application of knowledge in practice}

More than 1 in $5(22 \%, 19 / 86)$ of the participants describe being somewhat or not at all confident to recognise when MCAx is justified (figure 2). Furthermore, in response to a common clinical scenario considering a patient's capacity to refuse medical care, the majority of the participants $(77 \%, 64 / 83)$ reported previously experiencing this in practice. Over one-third $(35 \%, 29 / 83)$ of the participants self-rated as mostly competent; however, $15 \%$ (12/83) self-rated as not at all competent. Only onethird $(32 \%, 27 / 83)$ self-rated themselves as very competent $(29 \%, 24 / 83)$ or extremely competent $(4 \%, 3 / 83)$. 


\section{Figure 2}

Confident to recognise when mental capacity assessment (MCAx) is justified.

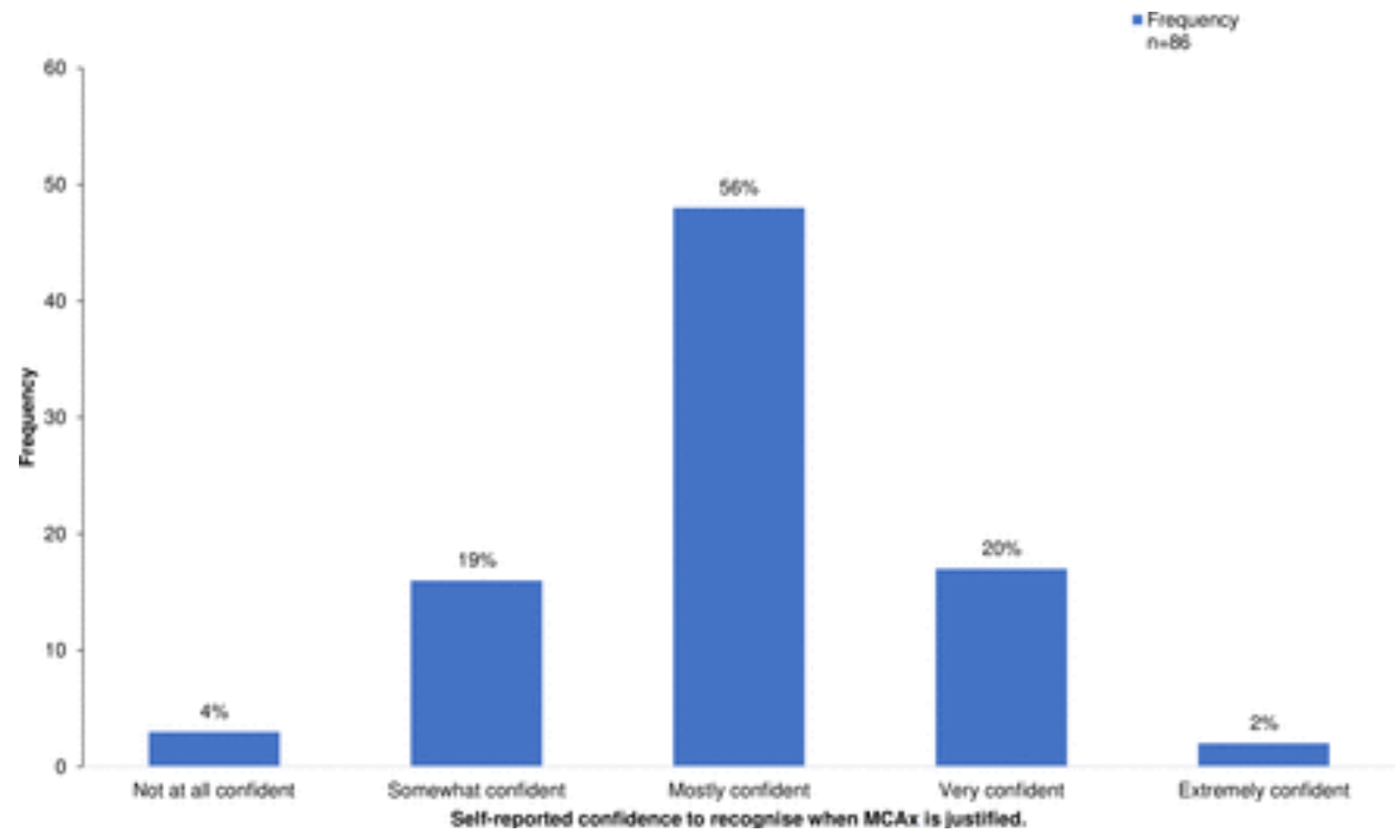

There was no evidence of a difference in MCAx scenario confidence by gender identity $(p=0.08)$ or country of primary medical qualification $(p=0.62)$. There however was a suggestion that self-reported confidence in performing MCAx in the scenario could be affected by career stage (figure 3 ). Secondary analysis was performed using the Kruskal-Wallis test which, after omitting the 'Other' career stage group of three doctors, indicated that there was evidence that doctors at varying career stages demonstrate different distributions of confidence scores $(p=0.001)$. Post hoc Bonferroni corrected pairwise comparisons, adjusting the $p$ values for multiple comparisons, showed that consultants with over 10 years of experience had significantly less confidence than early specialist training doctors $(p=0.007)$ and consultants with less than 10 years of experience $(p=0.039)$. 


\section{Figure 3}

Box-plot showing participant self-reported confidence in the given MCAx clinical vignette by career stage (Kruskal-Wallis Test $H(5)=18.40, p=0.002$ ). Confidence scale: 1 - not at all confident, 2 somewhat confident, 3 - mostly confident, 4 - very confident, 5 - extremely confident. MCAx, mental capacity assessment.
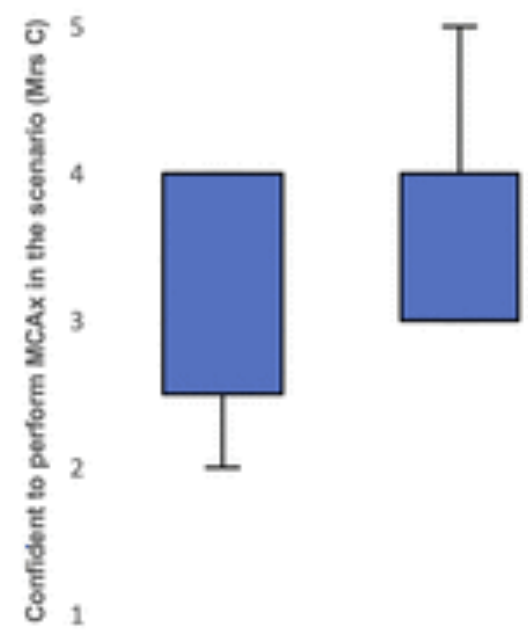

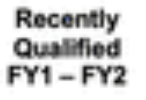

$n=5$
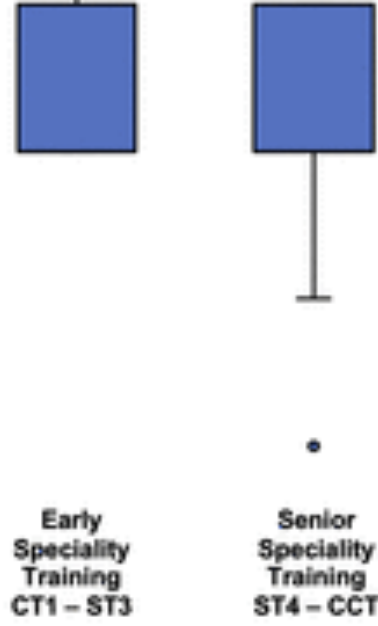

$n=11$
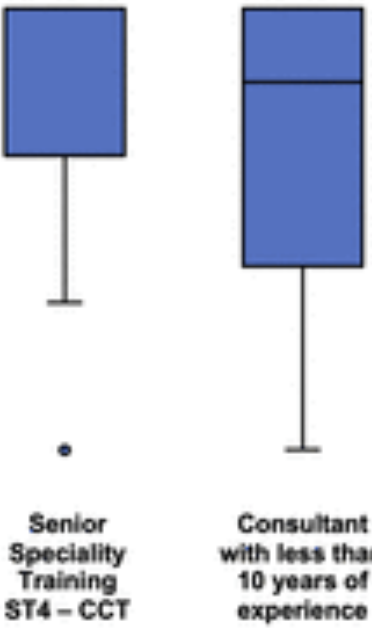

$\mathrm{n}=18$

$n=16$
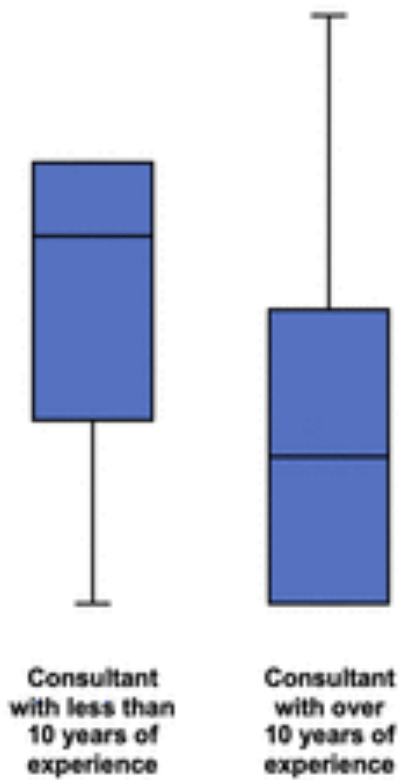

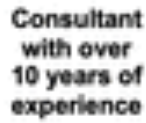

$n=31$

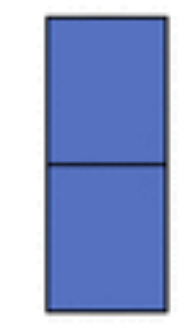

Other

nis

\section{Career Stage}

Further analysis of responses by the Mann-Whitney $U$ test with participant confidence compared by junior doctor and consultant career stage. The junior doctor group described significantly higher confidence compared with consultant career stage $(p=0.003)$, however, the median response between the two groups was 'mostly confident' (figure 4). 


\section{Figure 4}

Bar chart showing comparison between participant self-reported confidence in the given MCAx clinical vignette by career stage (Mann-Whitney $U$ test $p=0.003$ ). Confidence scale: 1 - not at all confident, 2 - somewhat confident, 3 - mostly confident, 4 - very confident, 5 - extremely confident. MCAx, mental capacity assessment.

\section{Juniors vs Consultants}

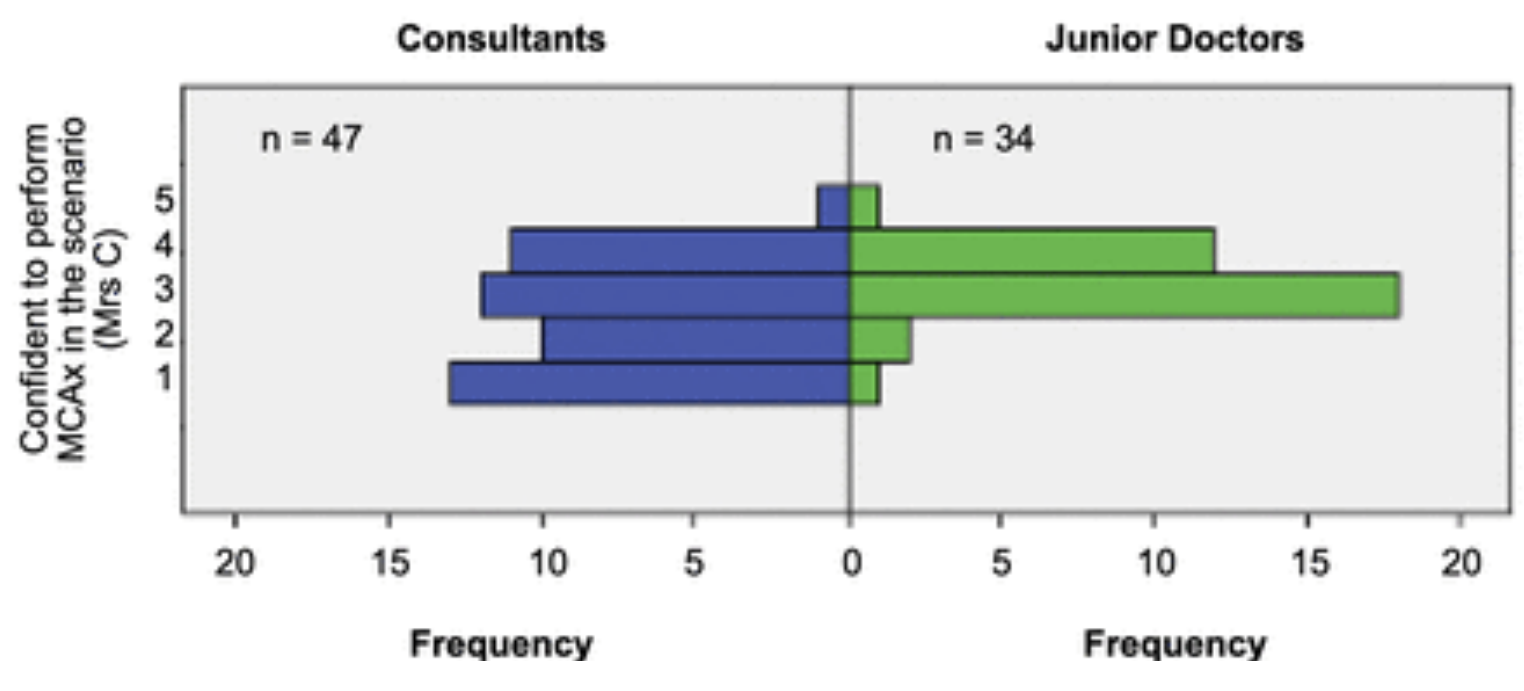

In consideration solely of their professional experience and disregarding the scenario, nearly half of the participants reported feeling under time pressure due to their workload $(45 \%, 41 / 92)$. Twelve per cent (11/92) of the participants also reported feeling under pressure or bullied by patients' relatives. Participants also reported experiencing a variety of feelings like anxiety $(33 \%, 30 / 92)$ and frustration $(21 \%, 19 / 92)$.

\section{Discussion}

This study provides quantitative data on previously unmeasured aspects of MCAx. Limited formal training and advancing career stage seem to demonstrate a negative effect on MCAx perception in doctors. It is clear that MCAx continues to be problematic for doctors and our findings are consistent with studies carried out prior to the introduction of the MCA. ${ }^{16} 17$ They also cohere with Shepherd and colleagues ${ }^{18}$ who showed that nearly half of the health and social care staff demonstrated consistently poor MCA knowledge.

In contrast to previous studies, doctors in this study reported better awareness of both the indications for performing MCAx and the key components which should be assessed. Despite being more aware of the legal requirements, we found evidence of the persistence of the findings of previous studies ${ }^{2}$ indicating that doctors, quite worryingly, do not practice in line with legislation. Our findings are also in keeping with Spencer and colleagues ${ }^{19}$ who directly observed doctors' and 
nurses' practice and found that assessments of decision-making capacity were sporadic and scantily documented. ${ }^{19}$

Why do doctors continue to report difficulty in applying their knowledge of mental capacity assessment in practice? Is it due to a lack of knowledge? Our findings demonstrate that despite limited formal training, most participants reported adequate knowledge of the components of mental capacity so presumably this knowledge was acquired from clinical experience. It could be that clinicians require more support in applying declarative knowledge about mental capacity in new situations so this transfers more readily to procedural knowledge. ${ }^{2021(\mathrm{p} .24)}$

When asked to describe their method of carrying out MCAx we found evidence to support unreliable performance of doctors with $15 \%$ admitting to incorrectly judging mental capacity against commonly held beliefs. Furthermore, $20 \%$ queried a MCAx due to concerns about the way in which the MCAx had been carried out. Our findings echo those of Spencer and colleagues ${ }^{19}$ and raise questions as to the way MCAx practice compares to other assessments, like physical examination and medical procedures. Would the reported variability in MCAx practice be acceptable in an assessment of a different clinical skill? Perhaps substandard clinical procedures are associated with higher perceived risk (and associated potential claims of negligence), whereas substandard MCAx procedure perhaps has lower perceived risk. The difference in attributable legal sequelae could lead to organisational cultures where less rigorous attention is paid to MCAx. Yet erroneously deeming a patient as lacking capacity can have far reaching consequences on that patient's autonomous decision-making wrongly denying them the basic right to accept or refuse care. ${ }^{22}$

The assessment of mental capacity is often the responsibility of senior members of the treating medical team. ${ }^{2(p .54)}$ It is therefore concerning to find that senior consultant participants in this study were least confident in performing MCAx. It is tempting to suggest that these findings reflect a historically casual approach to training doctors in capacity assessment in the context of a more paternalistically-based approach to practice. More prosaically, if these senior doctors are expected to carry out MCAx, but are actually lacking confidence, then this could be a contributing factor, reinforcing and perpetuating poor MCAx practice in junior colleagues.

Confidence may be described as 'the state of feeling certain about the truth of something,' or 'a feeling of self-assurance arising from an appreciation of one's own abilities or qualities. ${ }^{23}$ It is possible that experience can result in greater awareness of the consequences of the assessment leading to a more cautious approach. As the MCA is relatively new, senior consultants may 
appropriately perceive themselves to be less skilled at applying the principles of the Act. Conversely, doctors at earlier career stages may be better versed in the law but may be relatively overconfident novices and lack insight into the complexity of mental capacity assessment or their own abilities. ${ }^{24}$ The difference between novices and experts insight into their performance is well-established in other contexts so may partially explain our findings. ${ }^{25}$ However, additional factors such as the expectations of others and external influence of colleagues and patient relatives combined with time pressure could also undermine doctors' confidence to raise doubts. These factors may lead to fears of appearing less competent which could explain the anxiety experienced. However, this anxiety is not exclusive to senior Consultants as doctors from all career stages find MCAx difficult to the point of anxiety. The question of confidence in MCAx process prompts consideration of the way this process can be improved to better support doctors.

We were encouraged to find that half of the participants had queried the indication(s) for or outcome of a MCAx. This is perhaps arguably a positive marker of an institutional culture that prioritises safety so that doctors feel able to communicate freely with colleagues to minimise patient harm. ${ }^{26}$ However, it also reflects the complexity inherent in making decisions about mental capacity. The fact that doctors continue to encounter situations where MCAx is inappropriately considered leading to unnecessary assessments suggests that few doctors fully appreciate the scope of the legal basis for conducting MCAx as laid out in Section 2 (1) of the MCA. ${ }^{6}$

Doctors reported encountering situations where the decision to conduct a MCAx was inappropriate. This may reflect poor understanding of the triggers to assessment as set out in the MCA. ${ }^{6}$ The implications of such a deficit are that it makes it too easy for doctors to seek to use an erroneous finding lacking mental capacity to override a competent patient's apparently unwise decision despite this being a flagrant breach of the law. Future work to examine doctors' experiences of ambiguous capacity and consent decisions across a range of contexts seems justified.

When a mental capacity assessment was appropriately triggered the principal reason doctors reported for querying an assessment outcome concerned situations where a patient's capacity was fluctuating. This may suggest that mental capacity is perceived in a binary way despite the MCA's accompanying Code of Practice ${ }^{8}$ which requires doctors to regularly reassess a patient's capacity and to take steps to maximise each patient's ability to participate in decision-making. We suggest that future work addressing doctor experience of challenging situations in which capacity varies and of the trajectory of support for decision-making is needed. 
The MCA requires some consultation with those who know the patient best but the consultee does not determine the findings on decision-making capacity or any decisions made in the patient's best interests should they be determined to lack decision-making capacity. ${ }^{8}$ Therefore, it is concerning that doctors reported many instances where the outcome of a MCAx was queried in response to influence from colleagues and patient relatives. A balance should be struck between legitimate concern and excessive influence as it is evident that such influence can lead to inappropriate application of the MCA legal safeguards such as deprivations of liberty. ${ }^{8(p .110)}$ In light of the complexity and potentially significant impact on patients, the anxiety of doctors does appear to be justified.

This study has certain limitations. First, the sample was skewed to females but when compared with the most recent national data, gender identity distribution in this study is similar, with $58 \%$ of doctors in training and $47 \%$ of all UK doctors being female. ${ }^{27}$ Neurologists are the most represented speciality and may be more conversant with MCAx than doctors in other specialities leading to an under-identification of some issues. Despite the use of reminders the survey response rate of 106 out of 1027 was low; therefore, the results should be interpreted with some caution. We were reassured by the comparability between the demographic characteristics of the sample obtained and those of non-responders. However, if there was a selection bias, with doctors already interested in the topic taking the time to respond, then one could hypothesise that confidence and/or competence levels may be even lower in the general population of hospital doctors than in the sample obtained.

\section{Conclusion}

Effective and appropriate implementation of MCAx is fundamental to acceptable professional practice, but our findings suggest considerable variability in practice with many doctors lacking confidence in their ability to discharge their legal and professional responsibilities. Despite being aware of the law and professional best practice guidance there is significant variation in how doctors approach MCAx in practice. These findings coupled with doctors' self-reported lack of confidence in MCAx should be of concern to all healthcare practitioners.

\section{References}

1. Select Committee on the Mental Capacity Act 2005. Mental Capacity Act 2005: post-legislative scrutiny London2014 [Available from: https://publications.parliament.uk/pa/ld201314/ldselect/ldmentalcap/139/13902.htm.

2. Jayes M, Palmer R, Enderby $P$. An exploration of mental capacity assessment within acute hospital and intermediate care settings in England: a focus group study. Disability and Rehabilitation 2016:1-10. doi: https://doi.org/10.1080/09638288.2016.1224275 
3. Schofield C. Mental Capacity Act 2005 - what do doctors know? Medicine, science, and the law 2008;48(2):113-6. doi: https://doi.org/10.1258\%2Frsmmsl.48.2.113 [published Online First: 2008/06/07]

4. Emmett C, Poole M, Bond J, et al. Homeward bound or bound for a home? Assessing the capacity of dementia patients to make decisions about hospital discharge: Comparing practice with legal standards. International Journal of Law and Psychiatry 2013;36(1):73-82. doi: https://doi.org/10.1016/j.ijlp.2012.11.009 [published Online First: 2012/11/29]

5. Dunlop C, Sorinmade O. Embedding the Mental Capacity Act 2005 in clinical practice: an audit review. Psychiatr Bull (2014) 2014;38(6):291-93. doi: https://doi.org/10.1192/pb.bp.114.046870

6. Mental Capacity Act 2005. England and Wales, 2005.

7. The Law Commission. Mental Incapacity London: The Law Commission; 1995 [188-290]. Available from: http://www.bailii.org/ew/other/EWLC/1995/231.html231.

8. Department for Constitutional Affairs. Mental Capacity Act Code of Practice. London: The Stationery Office 2007.

9. Wilson E, Seymour JE, Perkins P. Working with the Mental Capacity Act: findings from specialist palliative and neurological care settings. Palliative Medicine 2010;24(4):396-402. doi: $10.1177 / 0269216309360739$

10. Manthorpe J, Samsi K. Care professionals' understanding of the new criminal offences created by the Mental Capacity Act 2005. International Journal of Geriatric Psychiatry 2015;30(4):384-92. doi: https://doi.org/10.1002/gps.4147 [published Online First: 2014/06/04]

11. Manthorpe J, Samsi K, Rapaport J. Dementia nurses' experience of the Mental Capacity Act 2005: A follow-up study. Dementia 2014;13(1):131-43. doi: 10.1177/1471301212454354

12. Bowling A. Research Methods in Health: Investigating Health and Health Services. 4th ed. Maidenhead, Berkshire, England: McGraw-Hill Education 2014.

13. Snow HA, Fleming BR. Consent, capacity and the right to say no. Medical Journal of Australia 2014;201(8):486-8. doi: 10.5694/mja13.10901

14. IBM Analytics. IBM SPSS Software Armonk, New York: IBM; 2017 [Available from: http://www.ibm.com/analytics/us/en/technology/spss/ accessed 8th January 2017.

15. ObjectPlanet I. Conduct Online Surveys using Opinio Oslo, Norway: ObjectPlanet, Inc.; 2017 [Available from: http://www.objectplanet.com/opinio/ accessed 7th January 2017.

16. Jackson E, Warner J. How much do doctors know about consent and capacity? Journal of the Royal Society of Medicine 2002;95(12):601-03. doi: 10.1258/jrsm.95.12.601 
17. Evans K, Warner J, Jackson E. How much do emergency healthcare workers know about capacity and consent? Emergency Medicine Journal 2007;24(6):391-93. doi: 10.1136/emj.2006.041293

18. Shepherd V, Griffith R, Sheehan M, et al. Healthcare professionals' understanding of the legislation governing research involving adults lacking mental capacity in England and Wales: a national survey. Journal of Medical Ethics 2018;44(9):632-37. doi: http://dx.doi.org/10.1136/medethics-2017-104722

19. Spencer BWJ, Wilson G, Okon-Rocha E, et al. Capacity in vacuo: an audit of decision-making capacity assessments in a liaison psychiatry service. BJPsych Bulletin 2017;41(1):7-11. doi: https://doi.org/10.1192/pb.bp.115.052613 [published Online First: 2017/02/12]

20. Schmidmaier R, Eiber S, Ebersbach R, et al. Learning the facts in medical school is not enough: which factors predict successful application of procedural knowledge in a laboratory setting? BMC Medical Education 2013;13(1):28. doi: 10.1186/1472-6920-13-28

21. Kaufman DM. Teaching and Learning in Medical Education

How Theory can Inform Practice. Third edition. ed. Hoboken, NJ: John Wiley \& Sons, Inc. 2019.

22. King JS, Moulton BW. Rethinking Informed Consent: The Case for Shared Medical DecisionMaking. American Journal of Law \& Medicine 2006;32(4):429-501. doi: https://doi.org/10.1177\%2F009885880603200401

23. Oxford Dictionaries. "confidence". Oxford Dictionaries: Oxford University Press; 2018 [Available from: https://premium.oxforddictionaries.com/definition/english/confidence.

24. Gude T, Finset A, Anvik T, et al. Do medical students and young physicians assess reliably their self-efficacy regarding communication skills? A prospective study from end of medical school until end of internship. BMC Medical Education 2017;17(1):107. doi: https://doi.org/10.1186/s12909017-0943-y [published Online First: 2017/07/02]

25. Sella F, Blakey R, Bang D, et al. Who gains more: Experts or novices? The benefits of interaction under numerical uncertainty. Journal of Experimental Psychology: Human Perception and Performance 2018;44(8):1228-39. doi: https://doi.org/10.1037/xhp0000526 [published Online First: 2018/07/27]

26. European Network for Patient Safety (EUNetPaS). Use of Patient Safety Culture Instruments and Recommendations Aarhus, Denmark: European Society for Quality in Healthcare - Office for Quality Indicators; 2010 [Available from: http://www.pasq.eu/DesktopModules/BlinkQuestionnaires/QFiles/448 WP4 REPORT\%20\%20 Use\%20of\%20\%20PSCl\%20and\%20recommandations\%20-\%20March\%20\%202010.pdf accessed 16 July 2019.

27. General Medical Council. The state of medical education and practice in the UK: 2017 London2017 [Available from: http://www.gmc-uk.org/somep2017. 


\section{Footnotes}

Acknowledgments The authors are grateful to all participants and to the Adult Safeguarding Team at University College London Hospitals. We are grateful for the support of Betsey Lau-Robinson, Head of Safeguarding Adults, the Mental Capacity Act \& Prevent; University College London Hospital NHS FT.

Contributors DP and JN conceived and designed the study. AL assisted with questionnaire development and implementation. DP disseminated the questionnaire, prepared the resulting data and conducted statistical analysis. AP advised on statistical analysis and the optimal reporting of results. DP drafted the manuscript, which was amended following comments from all other authors. All authors read and approved the submitted manuscript.

Funding The authors have not declared a specific grant for this research from any funding agency in the public, commercial or not-for-profit sectors.

Competing interests None declared.

Patient consent for publication Not required.

Ethics approval This study was registered with the UCL Data Protection Registration Service (Registration Number): Z6364106/2017/10/110. This project was reviewed by UCL's Research Ethics Committee (Project ID Number): $10453 / 002$ and was approved as a service evaluation.

Provenance and peer review Not commissioned; externally peer reviewed.

Data availability statement All data relevant to the study are included in the article or uploaded as supplementary information. 